\title{
Effect of the Sound Source Position Error on Distribution Characteristics of Underwater Shock Wave Bunching Sound Field
}

\author{
Xiaolong Liu $\mathbb{D}^{1},{ }^{1}$ Hongbing $\mathrm{Li}^{1,2} \mathrm{Ning} \mathrm{Li}^{3}$ and Kaizhuo Lei ${ }^{2}$ \\ ${ }^{1}$ Air and Missile Defense College, Air Force Engineering University, Xi'an 710051, Shaanxi, China \\ ${ }^{2}$ School of Marine Science and Technology, Northwestern Polytechnical University, Xi'an 710072, Shaanxi, China \\ ${ }^{3}$ Marine Design \& Research Institute of China, Shanghai 200011, China \\ Correspondence should be addressed to Xiaolong Liu; wusiguangchang2005@126.com
}

Received 28 April 2021; Accepted 7 July 2021; Published 15 July 2021

Academic Editor: Stanislav Vítek

Copyright $\odot 2021$ Xiaolong Liu et al. This is an open access article distributed under the Creative Commons Attribution License, which permits unrestricted use, distribution, and reproduction in any medium, provided the original work is properly cited.

Curved reflection bunching technique of underwater plasma sound source (UPSS) uses the geometric characteristics of the curved reflector to reflect and bunching intense sound shock wave, so the center position error of the sound source is one of the important factors affecting the bunching performance of the shock wave. In this paper, the cause of the sound source position error is analyzed in detail, and nonlinear finite element software ANSYS/LS-DYNA (dynamic analysis software developed by LSTC) is used to establish the model of the shock wave bunching sound field. Through numerical simulations, the shock wave bunching sound field distribution characteristics under the influence of different position errors are comprehensively simulated, and the bunching performance of the shock wave and its influence law are deeply analyzed according to the simulation results. It provides guidance for reasonably controlling the machining error and installation error of the reflector and discharge electrode, estimating the effective discharge times of the discharge electrode, and formulating the design process.

\section{Introduction}

UPSS technology can be used to realize narrow-pulse ultrawideband (UWB) sonar detection system, which breaks through the limitations of traditional sonar signal carrier modulation technology $[1,2]$. It has the advantages of high emission sound source level (up to $260 \mathrm{~dB}$ ), wide frequency bandwidth (bandwidth between tens of hertz and hundreds of kilohertz), high electroacoustic conversion efficiency, high distance resolution, strong penetration ability, and strong anti-interference ability [1-5]. At present, this technology has been widely used in civil fields such as extracorporeal shock wave lithotripsy, water treatment, oil pipeline blockage removal, rock fragmentation, and marine resources exploration. Especially in the fields of underwater target detection, underwater ultralong-range communication, and underwater acoustic countermeasures, it has become a hot research topic $[6,7]$.

The shock wave signal generated by UPSS arc discharge has weak directivity or no directivity, and the curved reflection bunching technology can be used to make the signal directional radiation and improve the radiation energy of the signal in the specified area. However, a series of "nonideal factors" will affect the sound field distribution of the shock wave in the process of bunching $[8,9]$, which makes it impossible to accurately describe the radiation directivity, bunching gain and focus position of the shock wave. As far as the system parameters of UPSS are concerned, the main "nonideal factors" include the structure and material of discharge electrodes, the distance between discharge electrodes, the structure and material of curved reflector, the central position of sound source, the type and capacitance of energy storage capacitor, and the discharge voltage. These factors will affect the parameters of shock wave generated by UPSS arc discharge. This paper focuses on the influence of the sound source position error on the sound field distribution characteristics.

Because the reflection bunching of shock wave is realized by the revolving ellipsoidal reflector, the center of discharge electrode should be located at the geometric focus of 
ellipsoidal reflector. But the structure of reflector and discharge electrode is relatively complex, processing error or installation error is easy to occur [10], and the electrode ablation is more serious under the condition of high-power repeated discharge. These factors will make the center position of the sound source deviate, thus greatly affecting the sound field distribution characteristics of the shock wave. In [10], 3D Max tool is used to analyze the influence of errors on the performance of ellipsoidal reflector bunching, including the processing error of reflector aperture and the position error of sound source. It is concluded that the reflector processing error has little influence on the focusing performance of the second focus, while the sound source position error has a great influence on the focusing performance. The research of reference [11] shows that as long as the first focus of the ellipsoidal reflector has a slight deviation, the pressure amplitude of the reflected wave in the focal area will change dramatically.

In this paper, the nonlinear finite element analysis method is used to establish the reflection bunching sound field model of shock wave. The influence of different sound source position errors, such as the machining error of electrode installation hole at the bottom of reflector, the machining and installation error of discharge electrode, and the ablation of discharge electrode, on the distribution characteristics of shock wave bunching sound field is analyzed by numerical simulation method. Therefore, the research in this paper has important practical significance for understanding the influence of source center position error on the characteristics of curved reflection bunching.

\section{Curved Reflection Bunching Technique of UPSS}

Acoustic lens method, curved reflection method, and array bunching method are commonly used in underwater acoustic engineering [12-17]. In this paper, the principle of ellipsoidal reflection bunching is used to realize the reflection bunching of shock wave generated by a single UPSS [6]. According to the linear acoustic theory, as shown in Figure 1, if the plasma sound source is placed on the first focus $F_{1}$ of the ellipsoidal reflector, the intense sound shock wave is reflected by the ellipsoidal reflector, and according to the geometric law of the ellipsoid, the energy will converge to the other focus $F_{2}$ of the ellipsoid. Therefore, the intensity of the bunching shock wave formed at the focus $F_{2}$ is obviously improved, the bunching gain is increased, and the pulse width of a shock wave is narrowed, so that the design requirements of a narrow-pulse UWB sonar detection system are met.

\section{Cause Analysis of the Sound Source Position Error}

As shown in Figure 1, when the ellipsoidal reflection bunching technique is adopted, the center position of the discharge electrode, i.e., the center position of the sound source, should be located on the first focus $F_{1}$ of the ellipsoidal reflector, and the discharge electrode structure is designed in a tip-tip type integrated way. When the discharge electrode mounting hole at the bottom of the ellipsoidal reflector has processing errors [10], i.e., the central axis direction of the mounting hole is inconsistent with the central symmetry axis direction of the reflector, the center position of the sound source will deviate from the first focal point $F_{1}$ of the reflector; when the processed discharge electrode tip-tip is not centered, the center position of the sound source deviates from the central symmetry axis of the reflector; when the discharge electrode is installed by direct insertion, the discharge electrode is not installed in place or exceeds the limit in the mechanical installation process. In this case, installation errors will occur, resulting in positive or negative movement of the sound source position in the central axis direction of the reflector. The sound source position is offset from that first focus.

In addition, copper alloy materials are often used as the electrode material, which belong to consumptive equipment in actual use. Under the condition of high-power and highfrequency discharge, the electrode is easy to ablate and wear, and the tip will be passivated, as shown in Figure 2. When the electrode tip is seriously passivated, the center position of the plasma sound source will shift.

Through the above analysis, we can divide the errors into the following two categories: (1) positive or negative position errors along the central symmetry axis of the reflector; (2) position error deviating from the central symmetry axis of the reflector.

\section{Modeling of the Shock Wave Bunching Sound Field and Error Analysis}

\subsection{Modeling of the Shock Wave Bunching Sound Field.} The distribution characteristics of the underwater plasma shock wave bunching sound field follow the three conservation laws of physics, so the basic control equations of the bunching sound field can be constructed by using the continuity equation, the motion equation, and the energy equation. The distribution parameters of sound field at different time and different position are solved [18]. Since there is almost no analytical solution for the equations, the arbitrary Lagrangian-Eulerian (ALE) method [19], which combines the advantages of Euler method and Lagrangian method, is usually used to solve the nonlinear bunching sound field distribution of high-power shock wave. This paper intends to study the influence of the sound source position error on the distribution characteristics of the shock wave bunching sound field, so in the simulation modeling, assuming that other simulation conditions remain unchanged, only changing the sound source position can meet the needs of numerical simulation. For ease of investigation, we directly use the nonlinear finite element analysis software ANSYS/LS-DYNA embedded with ALE algorithm to model and numerically simulate the bunching sound field [20].

The solid models of reflector, water medium, and discharge electrode are all divided by solid unit (SOLID164). In order to simulate the infinite free acoustic field, the boundary of the fluid computational domain is set as a nonreflecting boundary to eliminate the influence of 


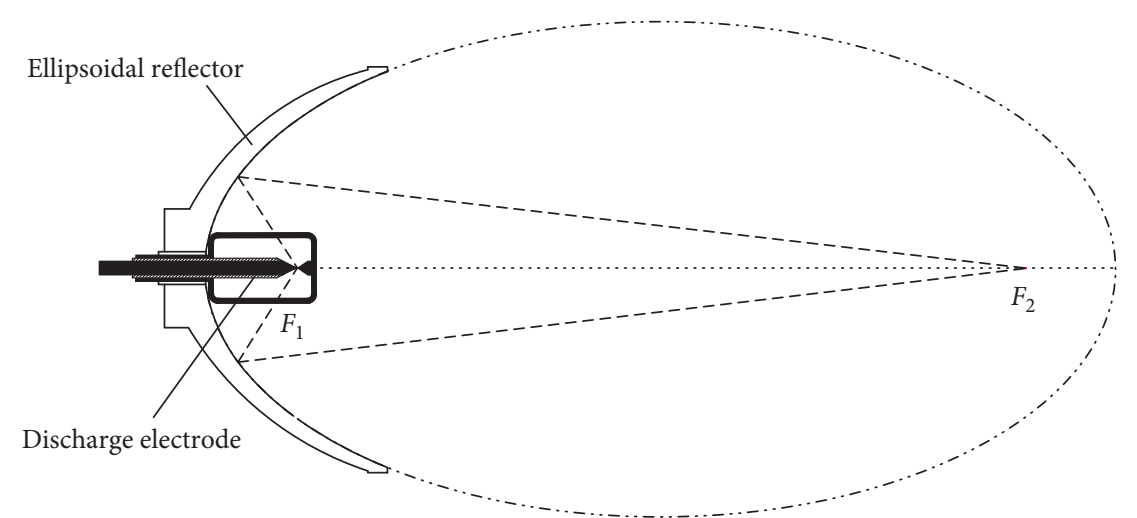

FIGURE 1: Schematic diagram of the shock wave reflection bunching principle based on the ellipsoidal reflector. $F_{1}$ is the first focus of the ellipsoidal reflector; $F_{2}$ is the other focus of the ellipsoid.

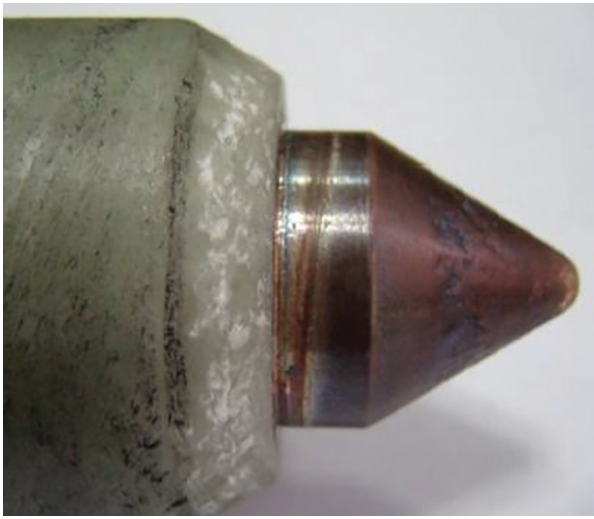

(a)

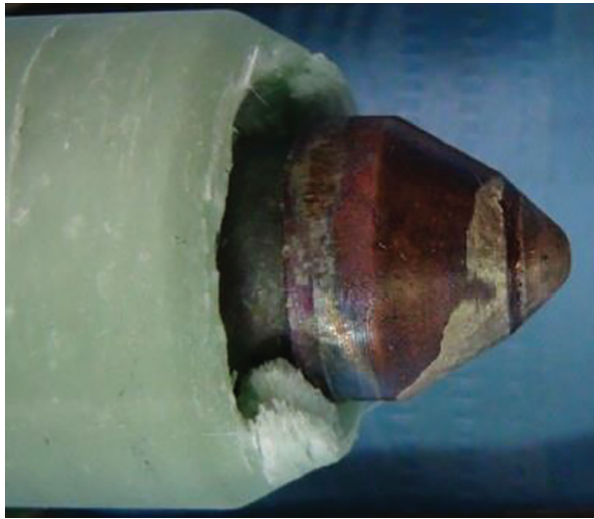

(b)

Figure 2: Ablation of the discharge electrode tip. Under the condition of high-power and high-frequency discharge, the electrode is easy to ablate and wear, and the tip will be passivated. (a) The electrode tip after hundreds of high-voltage pulse discharges. (b) The electrode tip after tens of thousands of high-voltage pulse discharges.

reflected waves. The contact among the ellipsoidal reflector, the discharge electrode, and the water medium adopts a common node method, and the multimaterial ALE algorithm is used among the three substances.

As shown in Figure 1, a hydroelastoplastic model JOHNSON-COOK is adopted for both that ellipsoidal reflector and the discharge electrode, and the equation of state is EOS_GRUNEISEN equation. The density of the reflector material is $0.001783 \mathrm{~g} / \mathrm{mm}^{3}$, and the discharge electrode material density is $0.0138 \mathrm{~g} / \mathrm{mm}^{3}$. The elastic-plastic model MAT_ELASTIC_PLASTIC_ HYDRO is used for the insulating material between the cathode and the anode of the discharge electrode. The compressible material model MAT_NULL is used for the water dielectric material; the equation of state is EOS_GRUNEISEN equation. The density of water is $0.001 \mathrm{~g} / \mathrm{mm}^{3}$, and the values of other parameters are $C=1484 \mathrm{~m} / \mathrm{s}, S 1=1.79, S 2=S 3=0, \gamma 0=0.11, a=3.0$. The calculation region is a region of $1200 \mathrm{~mm}$ in length, $500 \mathrm{~mm}$ in width, and $500 \mathrm{~mm}$ in height, the grid width is $1 \mathrm{~mm}$, and the calculation time is set to $1 \mathrm{~ms}$. The initial excitation waveform of UPSS is fitted by measuring the direct wave waveform at a point on the opening plane of the reflector, and the intense sound shock wave near the sound source is inversely deduced to load.

In order to verify the validity of the three-dimensional solid model established in this paper, taking the sound source center position $(0,90,0)$ as an example, the UPSS reflection bunching sound field distribution characteristics verification experiment is carried out. The experimental conditions are as follows: the energy storage capacitor is 5 $\mu F$, the distance between the electrodes is $4 \mathrm{~mm}$, the discharge voltage is $20 \mathrm{kV}$, the reflector is a rotating ellipsoid, the depth of the reflector is $180 \mathrm{~mm}$, the focal length is $1000 \mathrm{~mm}$, and the position of the sound source is the first focus with coordinates $(0,90,0)$. The shock wave is measured at three points on the sound axis, which are $240 \mathrm{~mm}$, $480 \mathrm{~mm}$, and $877 \mathrm{~mm}$ away from the center of the sound source, respectively, and the experimental measurement waveform and the simulation waveform are compared and analyzed, as shown in Figure 3.

It can be seen from Figure 3 that the experimental measurement waveforms at different measurement points on the sound axis are in good agreement with the simulation waveforms, which can better reflect the real situation of the 


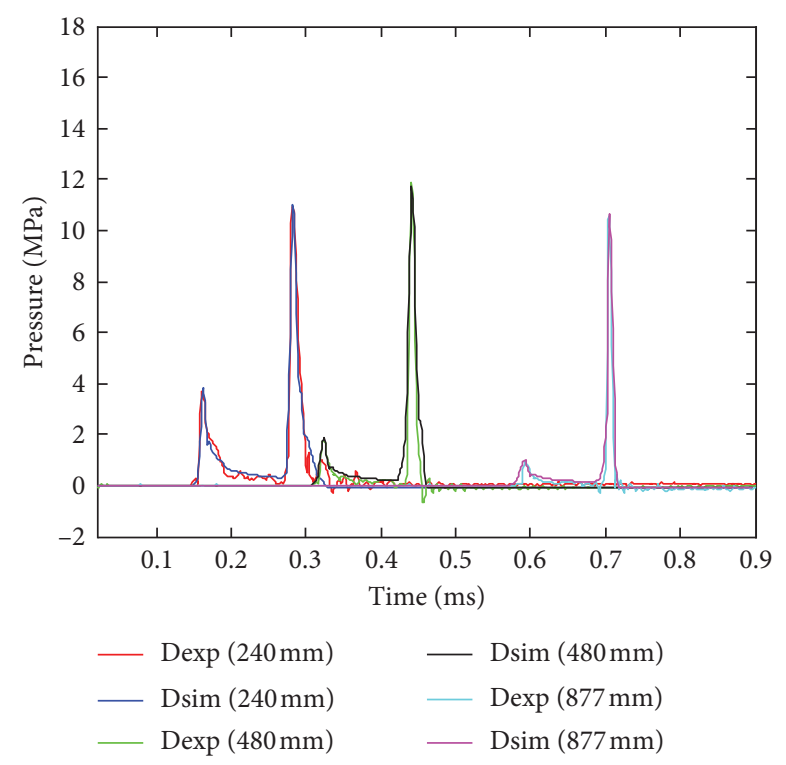

FIGURE 3: Comparison between simulation results and measured results. Dexp represents the experimentally measured waveform, and Dsim represents the simulated waveform.

bunching sound field. In the simulation waveform of Figure 3 , there is no negative value behind the bunching shock wave, which is mainly related to the setting of the pressure cutoff value of water, but does not affect the analysis of the bunching sound field distribution characteristics in this paper. Therefore, the bunching sound field model in this paper is effective.

4.2. Influence Analysis of the Sound Source Position Error. The g-mm-ms system is used in the numerical simulation. The depth of the ellipsoidal reflector is $180 \mathrm{~mm}$, and the center of the sound source (the first focus $F_{1}$ ) is $90 \mathrm{~mm}$. Here, we assume that the central symmetry axis of the reflector is the $Y$-axis, and the error in the direction of the $X$-axis or $Z$ axis deviating from the first focus $F_{1}$ is similar, so only the error in the direction of the $X$-axis is considered for the sound source position error deviating from the $Y$-axis. Therefore, only the errors along the $Y$-axis and the $X$-axis are analyzed in this paper, and the sound source position is set as $(0,90,0)$.

Firstly, the positive/negative position error generated in the $Y$-axis direction is analyzed. Assume that the direction propagating from the inside of the reflector to the outside along the $Y$-axis is the positive $Y$-axis direction, and the opposite direction is the negative $Y$-axis direction. We only analyze the error when the positive direction of $Y$-axis is $+2 \mathrm{~mm}$ and $+5 \mathrm{~mm}$, and the negative direction of $Y$-axis is $-2 \mathrm{~mm},-5 \mathrm{~mm}$. In order to facilitate comparative analysis, Figure 4 shows the effect of sound source position error along the $Y$-axis on the peak pressure of the bunching shock wave.

Figure 4(a) is the peak pressure distribution curve of the bunching shock wave in the $Y$-axis direction of the sound source, and it can be clearly seen that the peak pressure of the bunching shock wave when the error is $\pm 5 \mathrm{~mm}$ is obviously less than that of the other three cases, and the bunching effect is poor. For example, when the sound source position is $(0,85,0)$, the shock wave bunching effect is not obvious. Figures $4(\mathrm{~b})-4(\mathrm{~d})$ are the peak pressure distribution curves of the focal plane bunching shock wave at $775 \mathrm{~mm}, 887 \mathrm{~mm}$, and $1100 \mathrm{~mm}$ from the sound source center, respectively. When the error is $\pm 2 \mathrm{~mm}$, the peak pressure curve of the bunching shock wave is close to the normal situation, and it can also be seen that the maximum peak pressure of the bunching shock wave obtained by the positive error $+2 \mathrm{~mm}$ is greater than the negative error, and the focal plane area is smaller, and the peak pressure of bunching shock wave is higher.

It can also be seen from Figure 4(a) that when the error is $+2 \mathrm{~mm}$, the peak pressure of the bunching shock wave decreases rapidly after about $1000 \mathrm{~mm}$ from the center of the sound source, which is lower than the peak pressure of the bunching shock wave when there is no deviation. When the sound source position error in the $Y$-axis direction changes from the positive direction to the negative direction, the second focus position is moved along the $Y$-axis positive direction.

In order to further verify the reliability of the simulation results, we carried out experiments on the distribution characteristics of the bunching sound field based on different sound source positions. The experimental conditions are as follows: the sound source positions are $(0,70,0),(0,80,0)$, $(0.90,0)$, the first focus of the ellipsoidal reflector is located at $(0,90,0)$, the energy storage capacitor is $10 \mu \mathrm{F}$, the discharge voltage is $24 \mathrm{kV}$, the discharge electrode spacing is $4 \mathrm{~mm}$, and the experimental measurement point is the actual focus of the reflector. The distance from the first focus of the reflector is $1000 \mathrm{~mm}$. The measured waveform obtained at the same measurement point is shown in Figure 5.

It can be seen from Figure 5 that different sound source positions have obvious influence on the distribution of 

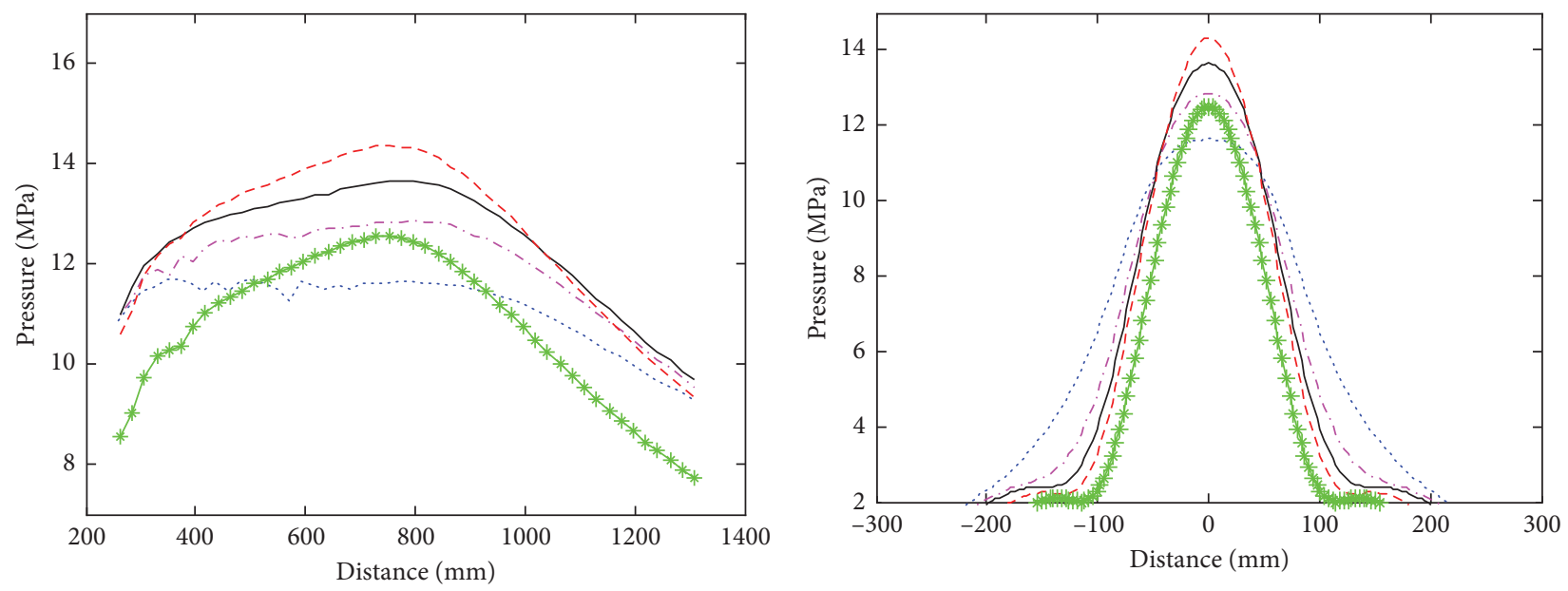

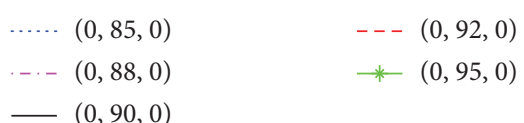

(a)

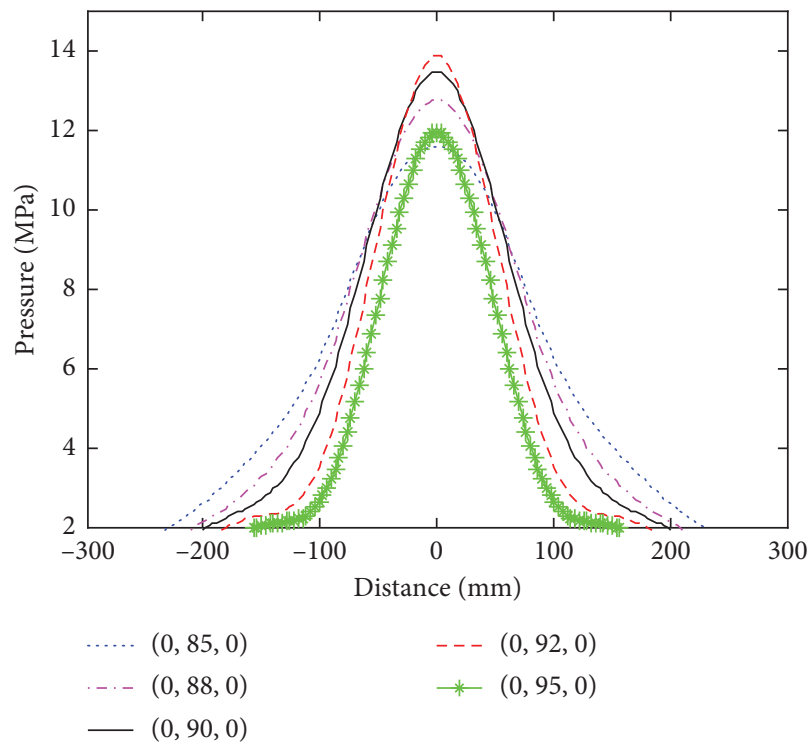

(c)

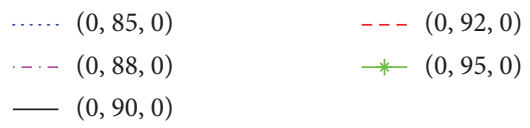

(b)

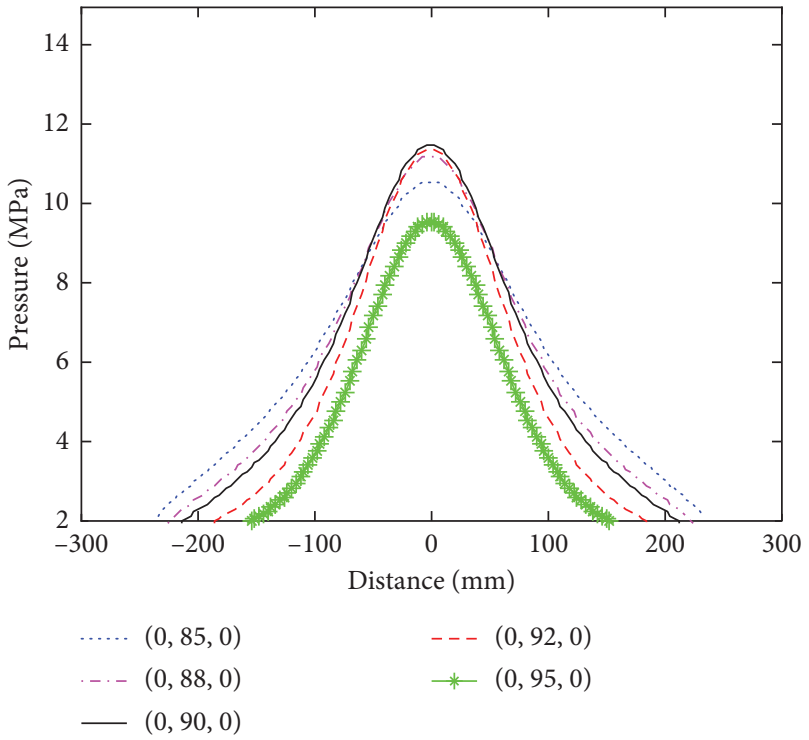

(d)

Figure 4: Influence of the sound source position error in the $Y$-axis direction on bunching characteristics. (a) The peak pressure distribution curve of the bunching shock wave in the $Y$-axis direction of the sound source. (b-d) The peak pressure distribution curves of the focal plane bunching shock wave at $775 \mathrm{~mm}, 887 \mathrm{~mm}$, and $1100 \mathrm{~mm}$ from the sound source center, respectively.

bunching sound field. When the direction of $Y$-axis changes from positive to negative, the waveform of bunching shock wave broadens and the bunching effect becomes worse. Especially when the sound source position is $(0,70,0)$, the bunching shock wave has two peaks, and the time delay between the bunching shock wave and the direct wave is the smallest, so the bunching effect is not obvious. This is consistent with the law of the influence of the source position error in the $Y$-axis direction on the bunching characteristics in Figure 4, which further illustrates the reliability of the simulation results in this paper.

Figure 6 shows the effect of sound source position error in the $X$-axis direction on the bunching characteristics, and
Figure 6(a) is the peak pressure distribution curve of the bunching shock wave on the central symmetry axis of the reflector. Figure 6(b) is the peak pressure distribution curve of the focused shock wave at the focal plane $1000 \mathrm{~mm}$ away from the center of the sound source, and Figures 6(c) and $6(d)$ are the bunching sound field distribution nephograms when the $X$-axis direction error is $+2 \mathrm{~mm}$ and $+5 \mathrm{~mm}$, respectively.

As can be seen from Figure 6, the influence of the sound source position error in the $X$-axis direction is larger than that in the $Y$-axis direction, and the larger the error, the worse the bunching effect. When the sound source position error changes in the positive direction of the $X$-axis, the 


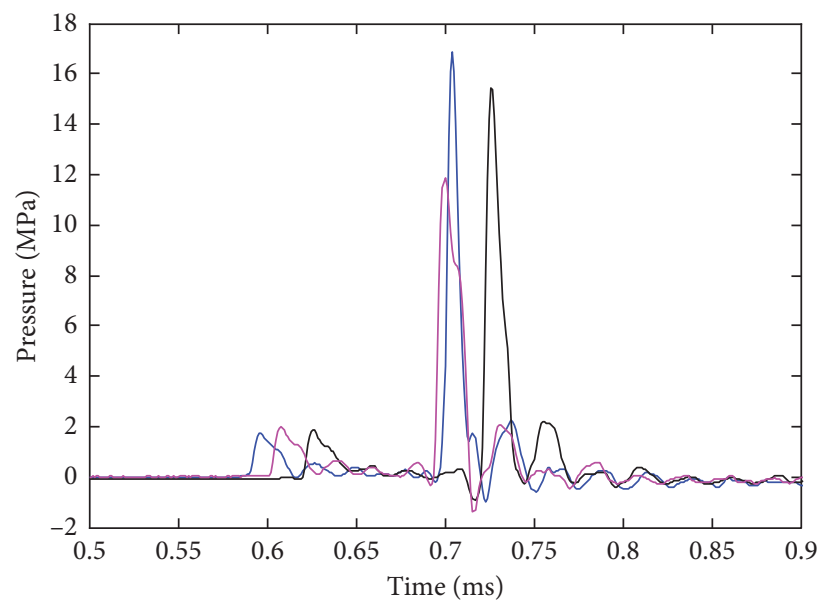

$-(0,90,0)$
$-\quad(0,80,0)$
$-\quad(0,70,0)$

FIGURE 5: Waveforms measured at the focal point under different sound source positions.
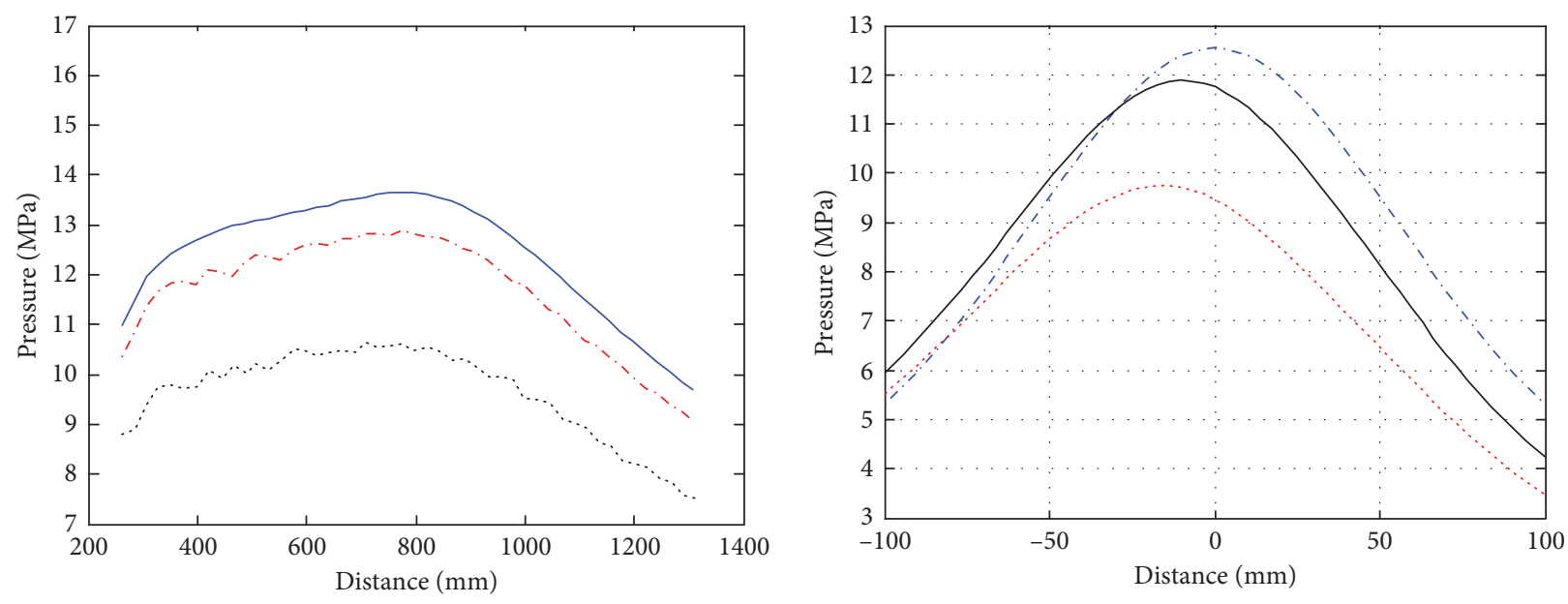

$-(0,90,0)$
$\ldots \quad(2,90,0)$
$\ldots \ldots(5,90,0)$

... $(0,90,0)$

- $(2,90,0)$

...... $(5,90,0)$

(a)

(b)

FIgURE 6: Continued. 


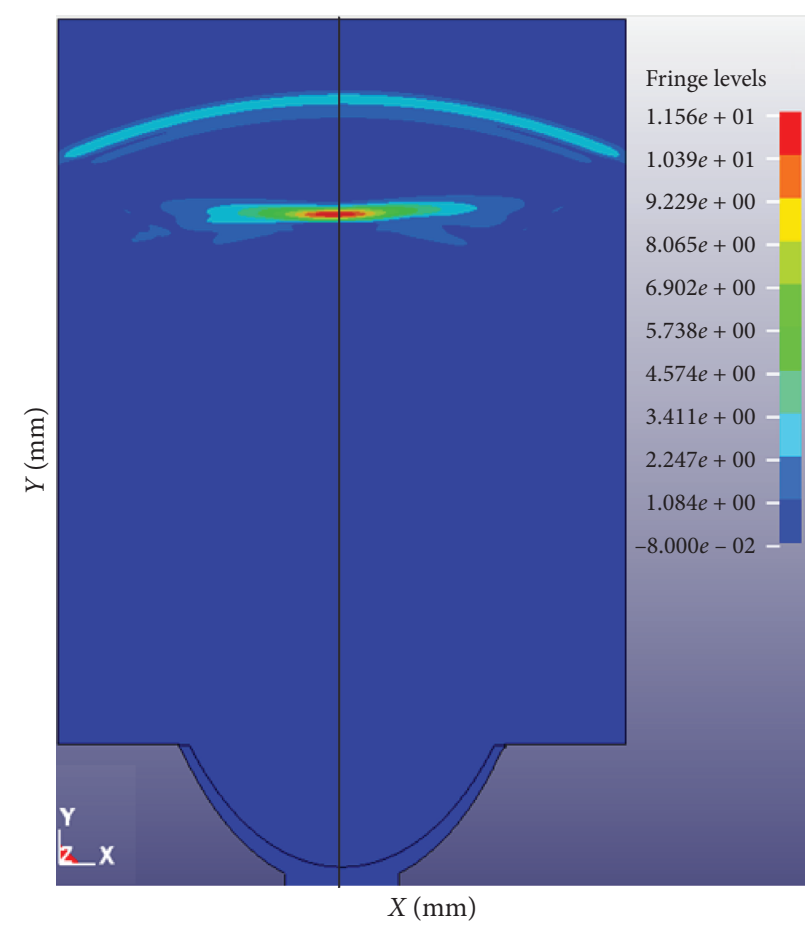

(c)

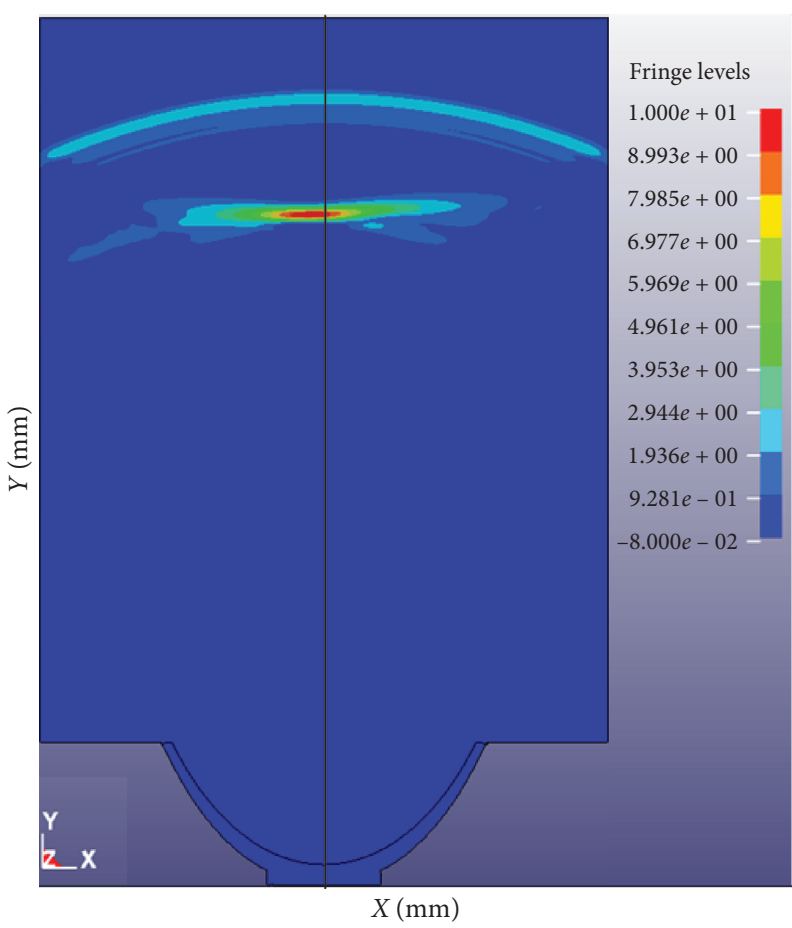

(d)

FIGURE 6: Influence of the sound source position error in the $X$-axis direction on bunching characteristics. (a) The peak pressure distribution curve of the bunching shock wave on the central symmetry axis of the reflector. (b) The peak pressure distribution curve of the focused shock wave at the focal plane $1000 \mathrm{~mm}$ away from the center of the sound source. (c, d) The bunching sound field distribution nephograms when the $X$-axis direction error is $+2 \mathrm{~mm}$ and $+5 \mathrm{~mm}$, respectively.

maximum shock wave peak pressure on the focal plane moves in the negative direction along the $X$-axis. Because the reflector is of an axisymmetric structure, the effects of sound source position errors in the $Z$ and $X$ axes are similar, so the effects of sound source position errors in the $Z$ axis are not discussed in this paper.

\section{Conclusions}

(1) There are three main reasons for the position error of the sound source: first, the machining error caused by the misalignment of the discharge electrode tip and the mounting hole at the bottom of the reflector; second, the discharge electrode mechanical installation errors; third, the discharge electrode ablation and passivation.

(2) When the position error in the direction of the central axis ( $Y$-axis) of the ellipsoidal reflector changes from the negative direction to the positive direction, the second focus position moves in the negative direction of the $Y$-axis. The positive and negative errors deviating from the central symmetry axis of the reflector will cause the focus position to move in the opposite direction.

(3) The position error in the direction deviating from the central symmetry axis of the reflector has a greater influence, while the position error in the direction of the central symmetry axis of the reflector has a smaller influence. Therefore, the machining error of the discharge electrode and the mounting hole should be strictly controlled, and the sharpness of the discharge electrode tip should be ensured, so as to reduce the error deviating from the central symmetry axis of the reflector. The negative installation error of the discharge electrode in the central symmetry axis direction of the reflector is greater than the positive error, and the control of the installation error of the discharge electrode can be determined according to the design requirements of the focus position and the peak pressure.

\section{Data Availability}

The data used to support the findings of this study are available from the corresponding author upon request.

\section{Conflicts of Interest}

The authors declare that they have no conflicts of interest.

\section{Acknowledgments}

This work was supported by the National Natural Science Foundation of China (no. 61701529) and Basic Research Project of Natural Science in Shaanxi Province (no. 2020JM353). 


\section{References}

[1] N. Li, J. F. Chen, J. G. Huang, and M. Fang, "Sounding mechanisms and characteristics of various underwater sound sources," Applied Acoustics, vol. 28, no. 4, p. 241, 2009.

[2] X. P. Lu, Y. Pan Huan, and H. H. Zhang, “水中脉冲放电的电 特性与声辐射特性研究, " Acta Physica Sinica, vol. 51, no. 7, p. $1549,2002$.

[3] R. Azevedo, P. Graneau, C. Millet, and N. Graneau, "Powerful water-plasma explosions," Physics Letters A, vol. 117, no. 2, pp. 101-105, 1986.

[4] A. Grinenko, Y. E. Krasik, S. Efimov, A. Fedotov, V. T. Gurovich, and V. I. Oreshkin, "Nanosecond time scale, high power electrical wire explosion in water," Physics of Plasmas, vol. 13, no. 4, p. 042701, 2006.

[5] S. Madhavan, P. M. Doiphode, and S. Chaturvedi, "Modeling of shock-wave generation in water by electrical discharges," IEEE Transactions on Plasma Science, vol. 28, no. 5, pp. 1552-1557, 2000.

[6] X. A. Feng, Y. F. Zheng, and Y. M. Zhang, "Water tank experiments of underwater target detection with high-power UWB pulse," Sciencepaper Online, vol. 3, p. 863, 2013.

[7] Q. P. Sun, X. B. Zhang, P. Yan, and Y. Cheng, Digital Ocean \& Underwater Warfare, vol. 3, no. 1, p. 24, 2020.

[8] Y. X. Mao, Study of influence of attenuation and non-linearity on focused sound fields, Master's Dissertation, University of Chinese Academy of Sciences, 2004.

[9] S. Y. Qian and J. W. Hu, "Research on nonlinear effects of sound fields induced by self-focused concave spherical transducers," Piezoelectrics \& Acoustooptics, vol. 29, no. 6, p. 720, 2007.

[10] T. Ma, J.-G. Huang, K.-Z. Lei, J.-F. Chen, and Q.-F. Zhang, "Simulating underwater plasma sound sources to evaluate focusing performance and analyze errors," Journal of Marine Science and Application, vol. 9, no. 1, pp. 75-80, 2010.

[11] R. O. Cleveland and J. A. Mcateer, "The physics of shock wave lithotripsy," Smith's Text Book of Endourology, BC Decker Inc, Hamilton, Canada, 2012.

[12] R. M. Yan, "On the focusing lows of spherical lens for ultrasonic testing," Technical Acoustics, vol. 13, no. 3, p. 124, 1994.

[13] A. Neisius, M. E. Lipkin, J. J. Rassweiler, P. Zhong, G. M. Preminger, and T. Knoll, "Shock wave lithotripsy: the new phoenix?" World Journal of Urology, vol. 33, no. 2, pp. 213-221, 2015.

[14] H. Z. Wang and S. Y. Qian, Journal of Shanghai Jiaotong University, vol. 30, no. 5, p. 109, 1996.

[15] H. Z. Wang, H. B. Yu, and P. Huang, Journal of Shanghai Jiaotong University, vol. 30, no. 1, p. 65, 1996.

[16] E. S. Ebbini and C. A. Cain, "A spherical-section ultrasound phased array applicator for deep localized hyperthermia," IEEE Transactions on Biomedical Engineering, vol. 38, no. 7, pp. 634-643, 1991.

[17] S. A. Goss, L. A. Frizzell, J. T. Kouzmanoff, J. M. Barich, and J. M. Yang, "Sparse random ultrasound phased array for focal surgery," IEEE Transactions on Ultrasonics, Ferroelectrics and Frequency Control, vol. 43, no. 6, pp. 1111-1121, 1996.

[18] J. H. Ferziger, Computational Methods for Fluid Dynamics, World Book Inc, Beijing, China, 3rd edition, 2012.

[19] J. G. Ning and T. B. Ma, Computational Explosion Mechanics, National Defense Industry Press, Beijing, China, 2015.

[20] H. S. Zhang, R. X. Hu, and S. T. Kang, ANSYS 14.5/LS-DYNA Nonlinear Finite Element Analysis Example Guide, China Machine Press, Beijing, China, 2013. 